\title{
Le système automatique d'information hydrologique (SAIH) en Espagne dans la gestion des crues fluviales. Expériences d'utilisation
}

\author{
Automatic system (SAIH) of hydrological information in Spain for river \\ flood management. Using experiences
}

par R. Espinosa

Confédération Hydrographique Sud de l'Espagne

\section{J. Yagüe}

Ministère Espagnol de l'Environnement

\section{R. Martinez}

Aminsa

Methods of river flood management in Spain have undergone a veritable revolution in recent years, from the progressive putting into operation of the SAIH (Automatic System of Hydrological Information) in the peninsular territory. The terrible episodes suffered in 1982 and 1983 marked a milestone in this sense, becoming the basic reason which generated the decision of the Spanish hydraulic administration to implant these systems. Designed from criteria of maximum technological innovation, the SAIH have fulfilled satisfactorily the objectives with which they were set up. Furthermore, they have grown into a real generator of development and innovation, both in the hydraulic administration itself, of which they form part, as well as in the rest of the bodies involved in the management of river flood situations, the ultimate purpose of which is the reduction of potential damage.

In this paper, the main characteristics of these systems are described, they are fitted into the administrative and territorial frame with which they relate and, as an example, some real events that have occurred are described.

\section{INTRODUCTION}

1.1 Cadres administratif, législatif et territorial

Actuellement, l'action en situation de crue sur le territoire national espagnol, relevant de l'Etat, s'articule autour des principales compétences attribuées aux ministères de l'Environnement (MIMA : Ministerio de Medio Ambiente) et de l'Intérieur (MI). Le premier abrite les services chargés de l'information en matière d'hydrologie et de météorologie. Du second, le MI, dépendent les services de Protection
Civile (PC), chargés du secours de la population et de la coordination des moyens matériels et humains disponibles.

En Espagne, l'administration compétente en matière d'hydrologie est du ressort des organismes appelés Confédérations Hydrographiques $(\mathrm{CCHH}$, au nombre de 10 sur le territoire péninsulaire espagnol). Ces confédérations agissent dans leurs domaines de compétence respectifs, organisés en fonction des principaux bassins hydrographiques de la péninsule.

A leur tour, les $\mathrm{CCHH}$ dépendent de la Direction Générale des Travaux Hydrauliques (DGOH : Dirección General de Obras Hidráulicas), qui assure la coordination et l'action 


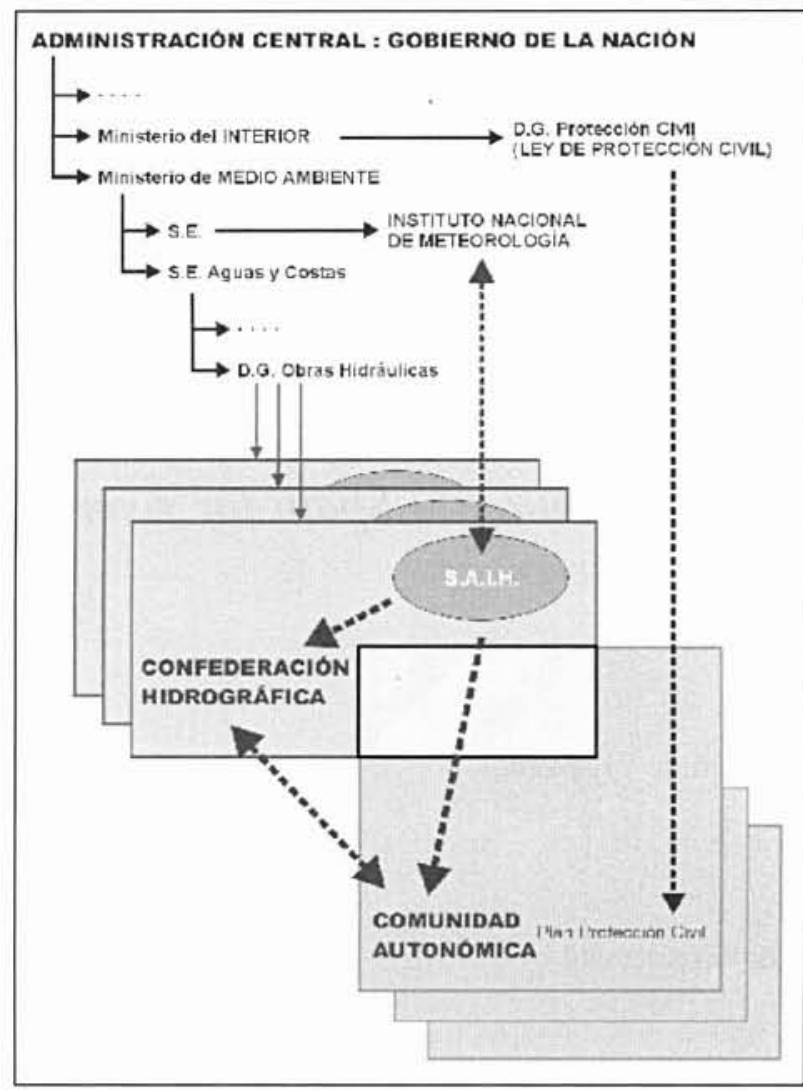

1. Schéma administratif.

au niveau national et qui relève du MIMA, à travers le Secrétariat d'Etat des Eaux et des Côtes.

Du point de vue législatif, l'administration hydraulique est régie par la Loi des Eaux de 1985 et les Règlements postérieurs qui la complètent. Ceux-ci spécifient les domaines d'action propres à chaque $\mathrm{CCHH}$, ainsi que la composition organique des services intégrant chaque CCHH. La Loi mentionne également certains aspects intéressants pour le sujet qui nous occupe. Notamment, elle confère aux $\mathrm{CCHH}$ certaines attributions et missions telles que la réalisation de statistiques et le contrôle de l'hydrologie, ainsi que l'information sur les crues, en consultation avec l'Administration centrale, les Communautés Autonomes, les corporations locales, etc. Elle définit aussi la figure du Comité Permanent pour des situations exceptionnelles, chargé de réaliser la gestion hydraulique le cas échéant, et servant de liaison avec les services de Protection Civile.

Dans les situations de crue, la référence principale de type législatif est celle qu'établit la « Directive Fondamentale de Planification de la Protection Civile devant le Risque d'Inondations » (DBPPCRI), élaborée par la Direction Générale de Protection Civile (DGPC) au nom du ministère de 1'Intérieur.

Entre autres, cette directive établit la structure générale de cette planification conformément à la subdivision territoriale et administrative qui existe dans notre pays. Elle établit aussi la composition du Comité National de Coordination dont font partie, en tant que membres, le Directeur Général des Travaux Hydrauliques (DGOH) et celui de l'Institut National de Météorologie (INM), dépendant tous les deux du MIMA. Elle définit encore le Système de Prévision et d'Information Hydrologique (SPIH), rassemblant les services, moyens et ressources de la DGOH et des $\mathrm{CCHH}$.

La structure de l'administration territoriale espagnole conditionne également ce schéma : l'Espagne est formée d'une agrégation de "Communautés Autonomes " (CCAA, 15 sur la péninsule), délimitées selon un critère de compartimentation régionale, et qui assument des compétences importantes et nombreuses dans leurs circonscriptions respectives.

Le Plan national de Protection Civile contre les inondations impose d'élaborer des plans similaires au niveau des Communautés Autonomes ; ce sont eux qui déterminent réellement l'action et la prévision en situation d'urgence provoquée par les crues.

Pour résumer tout ce panorama, nous pourrions évoquer une cellule de coordination et d'opération en situations de crues sur un territoire donné, composée de trois éléments fondamentaux : 1) les $\mathrm{CCHH}$, chargées de fournir l'information hydroclimatique et les prévisions, et responsables des opérations de la structure hydraulique ; 2) le SAIH, dont traite cette communication, investi d'une mission d'information et de prévision au sein de chaque $\mathrm{CCHH}$; et 3) la Communauté Autonome, chargée de l'exécution des plans respectifs de Protection Civile, de la coordination des moyens d'action, et responsable de la diffusion des informations pertinentes.

\subsection{Hydrologie péninsulaire et extrême}

L'Espagne est un pays à l'hydrologie difficile, due à la grande variabilité dans l'espace et dans le temps qui caractérise son régime de pluviométrie. Avec une pluviométrie moyenne qui se situe entre 500 et $600 \mathrm{~mm} / \mathrm{an}$, coexistent des zones enregistrant des valeurs moyennes supérieures à $1000 \mathrm{~mm} / \mathrm{an}$ (étroite bande côtière au nord de la péninsule et quelques parcelles dans le centre et au sud-ouest), soumises à l'influence atlantique, avec d'autres de type désertique avec des quantités inférieures à $200 \mathrm{~mm} / \mathrm{an}$ (sud-est), caractéristiques du régime méditerranéen. En outre, le pays connaît fréquemment de longues périodes consécutives de sécheresse entrecoupées d'années humides, de sorte que les valeurs moyennes sont largement dépassées.

L'historique des situations de crues catastrophiques que nous avons connues révèle néanmoins un fait notable, caractéristique de ce clivage climatique. La majorité de ces situations se produisent dans les zones à plus petite pluviométrie annuelle, comme il s'en avère quand on considère, par exemple, les inondations qui ont eu lieu au cours des 40 dernières années et ont causé la mort de 1600 personnes (soit une moyenne de 40 victimes par an), ainsi que des dégâts matériels atteignant près de 300 millions d'euros par an. D'après les chroniques, 2400 épisodes d'inondation seraient attestés pour les 500 dernières années, ce qui signifie une moyenne appréciable de 5 situations par an.

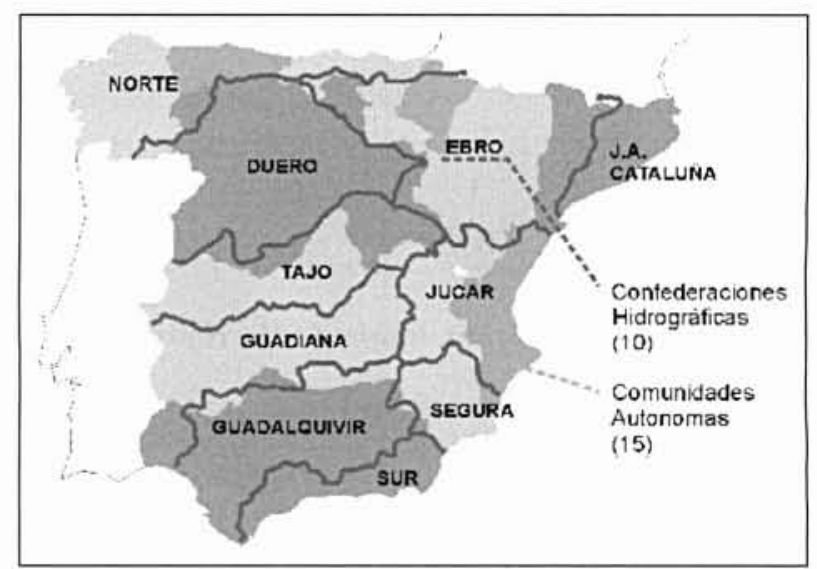

2. Schéma territorial. 


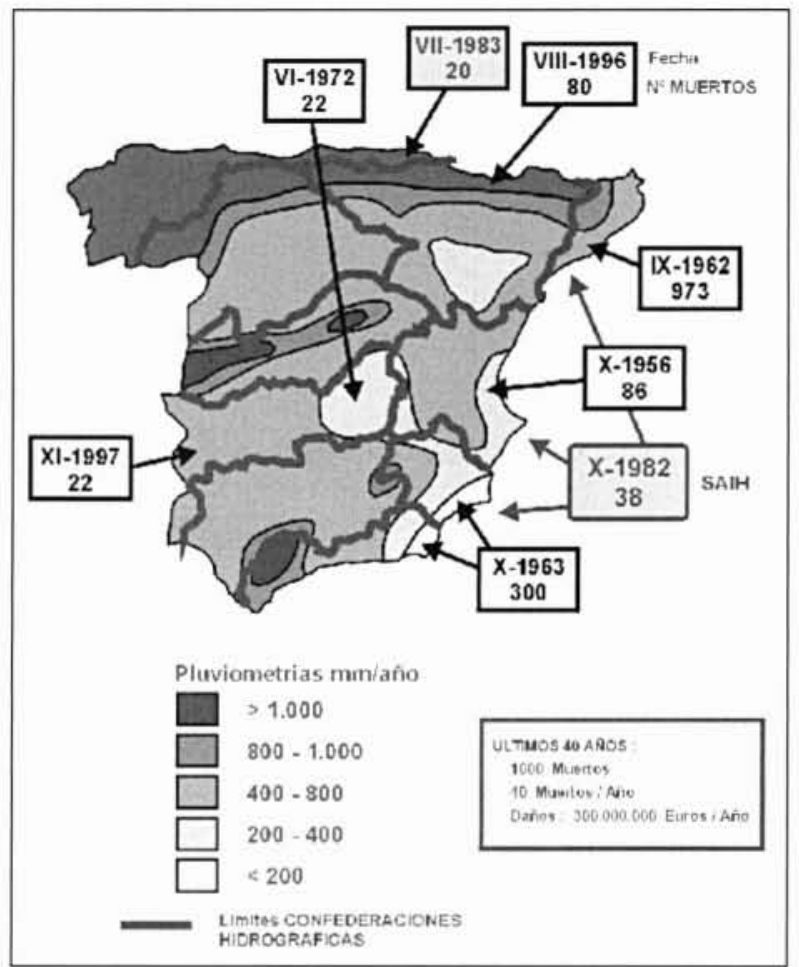

3. Hydrologie et inondations.

\section{II — LE PROGRAMME SAIH}

\subsection{Définition et objectifs}

Face à ce tableau, l'administration espagnole décide en 1984 , juste après les graves inondations de 1982 (causant la rupture du barrage de Tous) et de 1983, d'implanter les systèmes SAIH de contrôle des bassins sur tout le territoire péninsulaire. La description des activités de la première phase, centrée sur l'analyse de solutions alternatives et l'adoption de critères de conception, serait très longue à expliquer dans le cadre de cette communication. En bref, on relèvera la mise en cuvre de trois objectifs directs fondamentaux : 1) Déclenchement et suivi des alertes en cas de crues ; 2) Gestion quotidienne des ressources hydriques et infrastructures associées; et 3) Contrôle de la sécurité des barrages.

La conception des systèmes SAIH permet en plus l'incorporation d'objectifs supplémentaires de grande importance, comme : 4) la modernisation des procédures de gestion des $\mathrm{CCHH}, 5)$ l'amélioration de la structure, consistance et fiabilité des bases de données, 6) la possibilité d'incorporer des mécanismes de contrôle à distance et de télécommande, 7) le contrôle de la qualité des eaux superficielles et souterraines, etc.

La conception des systèmes SAIH répond à une série de critères bien définis au préalable :

- Décentralisé au niveau national. Chaque $\mathrm{CCHH}$ possède son propre système, indépendant des autres. La DGOH assume un rôle de coordinateur entre les divers SAIH. Dans chaque $\mathrm{CCHH}$, le système est fondamentalement centralisé, et l'on dispose d'un centre de contrôle au siège principal de la $\mathrm{CCHH}$.

- Automatique, totalement en ce qui concerne la mesure à distance, et fonctionnement général permanent nécessitant le moins d'assistance possible, capable de générer des signaux d'alerte dans tous les cas critiques prévisibles.

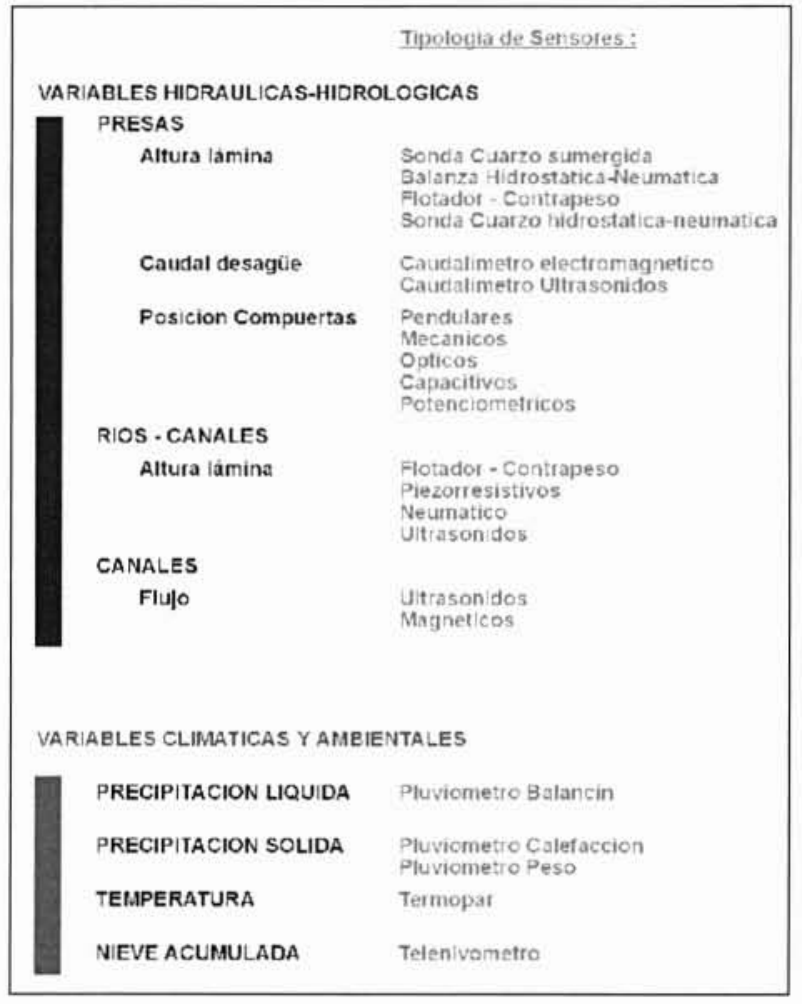

4. Typologie Capteurs principaux.

- Fonctionnant en temps réel, de façon à permettre une connaissance immédiate de la réalité hydro-météorologique, ainsi que de l'évolution prévue. Dans la plupart des systèmes SAIH, on a cherché à réduire le temps d'arrivage de l'information au minimum, environ 15 minutes, ce qu'on considère un temps réel par rapport au temps que prennent à se produire les phénomènes qu'on prétend contrôler.

- Robuste, afin d'assurer le moins de failles possible dans tous et chacun de ses éléments constitutifs et de réduire au maximum les travaux correctifs et préventifs de maintenance ; autonome quant à l'alimentation en énergie et au fonctionnement des communications.

- Permettant de connaître les processus avec un maximum d'anticipation, ce qui conditionne la topologie des réseaux implantés, des systèmes de communications et la typologie des applications utilisées pour confectionner les données.

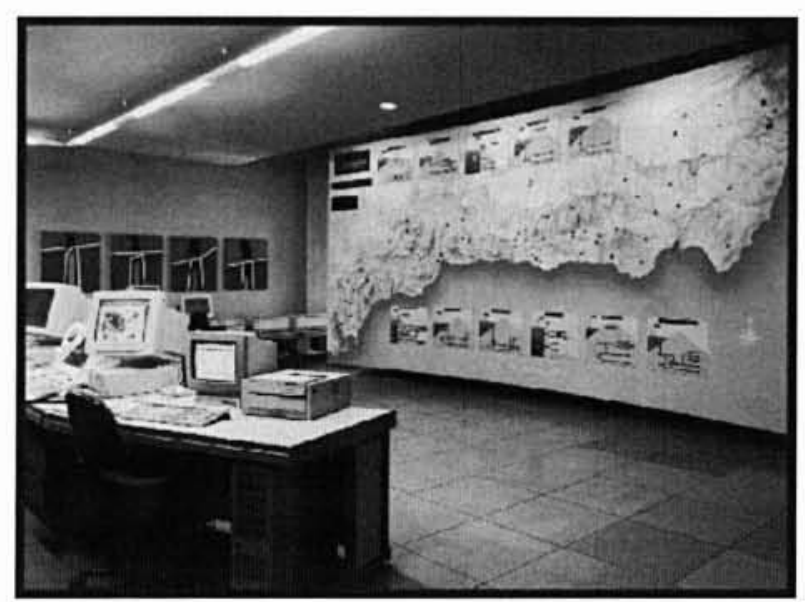

5. Centre de contrôle (SAIH Sur). 


\subsection{Eléments du système}

Dans sa structure ordinaire, chaque SAIH se compose de trois éléments principaux :

- un réseau de postes d'observation, qui, grâce à des capteurs, recueillent l'information prête à être transmise ;

- un réseau de communications, chargé de dispenser l'information captée dans le centre de traitement correspondant. Actuellement, à côté des systèmes qui effectuent cette transmission par radio terrestre (les plus anciens), il existe des systèmes qui fonctionnent par satellite ou de façon mixte ;

- un centre de contrôle, où l'on rassemble toute l'information recueillie et où on effectue des opérations de contrôle, élaboration, stockage, distribution, interprétation, diagnostic et prise de décisions. Soulignons au passage le haut degré de technologie atteint dans les techniques de modélisation hydrologique employées, qui comprennent une large gamme de méthodes s'appuyant sur des concepts très variés, avec des modèles de type déterministe et stochastique, et qui opèrent dans des environnements mis au point selon les techniques utilisées dans les systèmes experts fondés sur le fonctionnement de l'intelligence artificielle, et d'autres basés sur le développement des réseaux neuronaux capables d'auto-apprentissage.
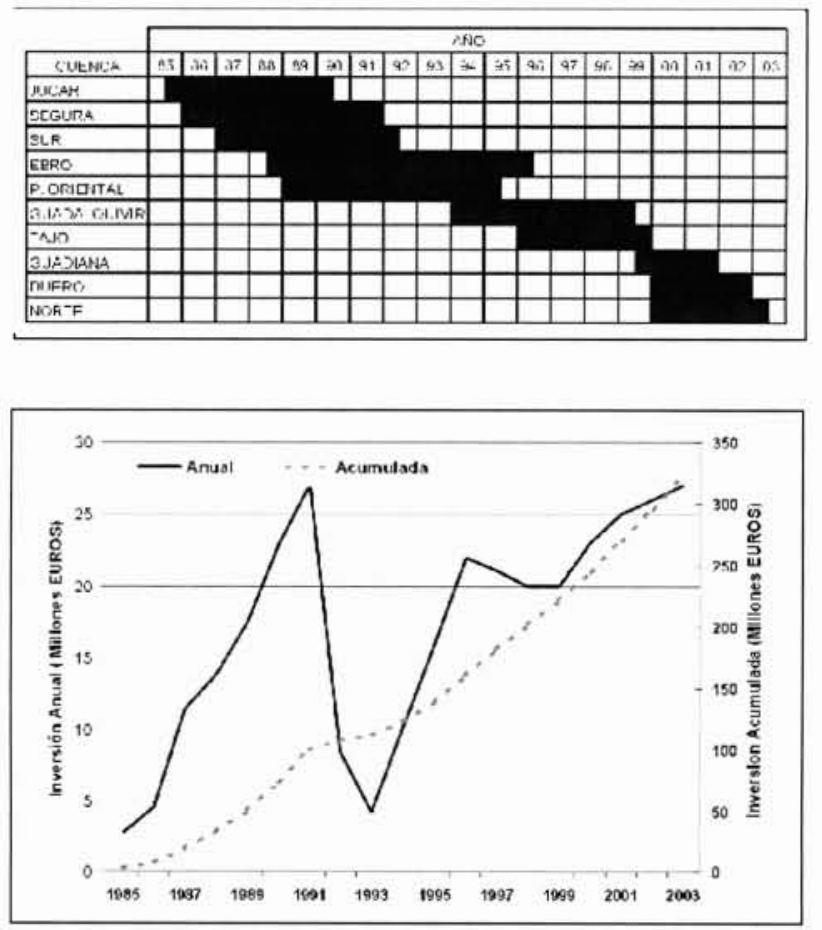

\section{Programmation et investissement SAIH.}

\subsection{Programme d'implantation}

L'implantation du SAIH a commencé dans le milieu de l'année 1985, et c'est le domaine de la CCHH du Júcar qui a été équipé le premier. Plus tard, on a installé et mis en marche les SAIH correspondant aux CCHH du Segura, du Sud, de l'Ebre et des Bassins de Catalogne. Celui du Guadalquivir est pratiquement terminé et celui du Tage est déjà bien avancé. On vient d'entreprendre les premières phases du contrat du SAIH du Guadiana, et l'on prévoit d'entamer les travaux pour le Nord et le Douro en l'an 2000 .

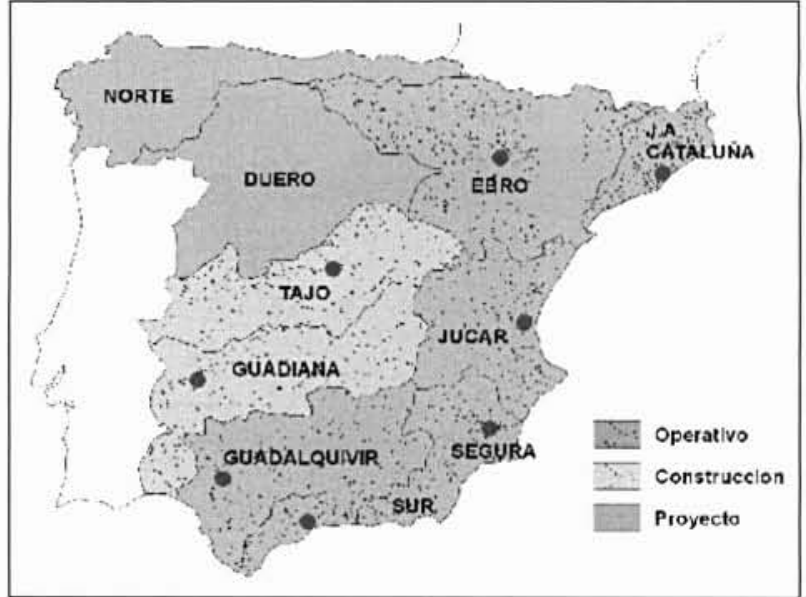

7. Etat d'implantation SAIH.

Ainsi donc (SAIH allant du Júcar au Guadiana), on dénombre actuellement un total de $386000 \mathrm{~km}^{2}$ de surface contrôlée (pour un total de $500000 \mathrm{~km}^{2}$ de la Péninsule); à cet effet, on utilise un total de 1421 points de contrôle munis de capteurs, dont 322 implantés dans les barrages, 388 dans les rivières, 224 dans les canaux, 338 pluviomètres et 218 répéteurs de radio.

Tout cela a supposé un investissement de 225 millions d'Euros, investissement qui se chiffrera à 325 millions quand on aura fini d'équiper toute la péninsule.

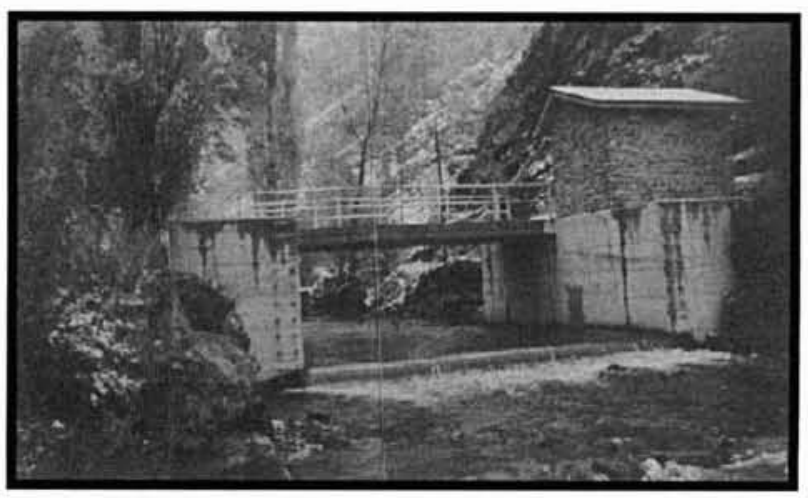

8. Station de jaugeage.

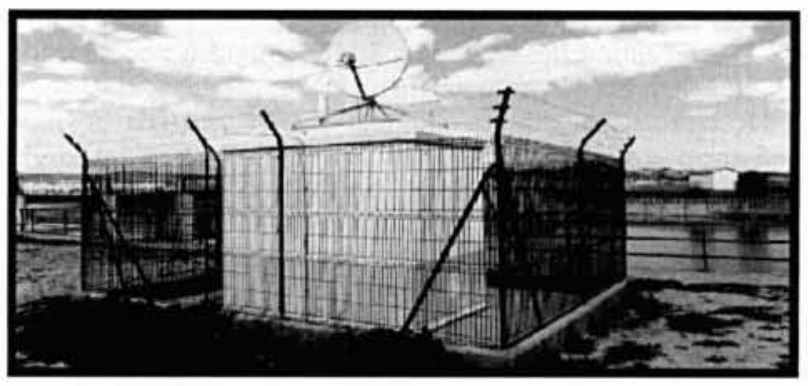

9. Station lointaine Vsat. 


\section{EXPÉRIENCES D'UTILISATION. APPLICATIONS HYDROLOGIQUES DES SAIH}

\subsection{Gestion des crues : coordination entre les institu- tions}

La prestation générique d'un SAIH dans les situations de risque d'origine hydro-météorologique, c'est de fournir des informations, au sens de ce qui permet de réduire l'incertitude. Sous cette étiquette générale, on peut distinguer deux catégories de produits ou services.

D'une part, il y a des produits directement générés par les $\mathrm{SAIH}$ en tant que "machines à information ", comme l'information hydrologique directe (essentiellement pluviométrie ponctuelle et niveaux des cours d'eau et bassins) qui permet d'établir l'état hydrologique actuel du bassin et son évolution récente. L'utilité de cette information vient du fait qu'elle est très actuelle, étant donné qu'on travaille en temps réel - ou presque.

Au surplus, les SAIH génèrent un autre type d'information plus élaborée, de qualité et complexité croissant à mesure que se consolident les systèmes, et qui permet d'estimer, en fonction de la taille des bassins, leur évolution immédiate (nowcast) ou à long terme (forecast).

Mais par ailleurs, d'autres prestations sont apparues, qui vont bien au-delà des prestations relatives à la dimension hydrologique du processus, et qui sont du domaine opérationnel. Outre la supervision permanente exercée par les centres de contrôle SAIH, ces derniers se sont établis spontanément dans des centres qui rassemblent toute l'information de type hydro-météorologique, indépendamment des sources génératrices. Cette centralisation de l'information en un seul endroit, ainsi que le haut niveau de spécialisation atteint par ces services, ont eu un effet synergique pour la compilation, comparaison, intégration et interprétation des données et informations vers les usagers terminaux, particulièrement les services de protection civile et les gestionnaires de barrages.

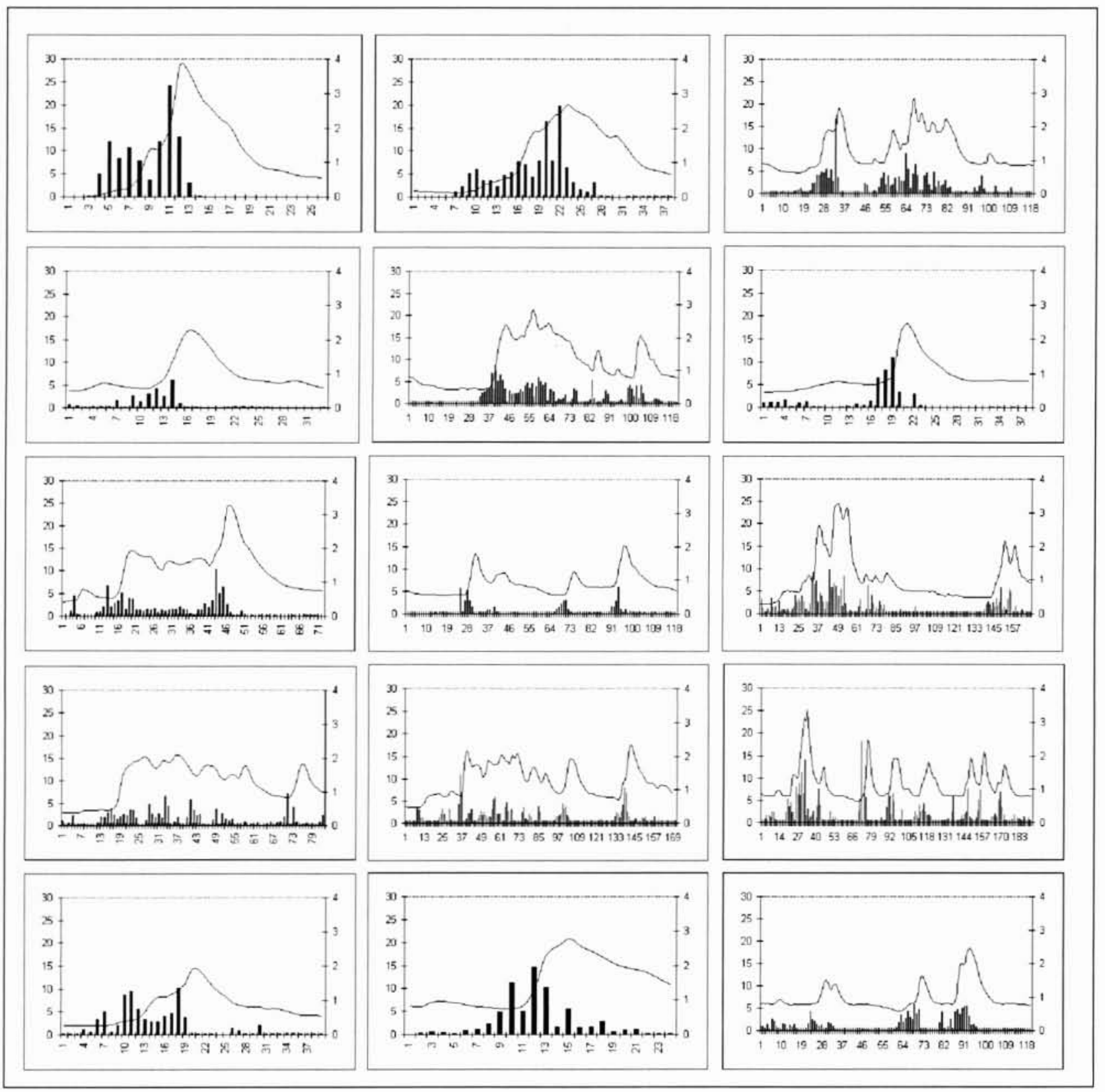

10. Principales crues enregistrées par le réseau SAIH dans l’Hozgarganta. Période décembre 1995 - février 1998. 
Ces circonstances ont donné lieu à la création d'un scénario inédit et sans comparaison avec le précédent, dans lequel la dispersion des sources favorisait la génération chaotique d'informations, souvent déformées et contradictoires, ce qui occasionnait une perte de contrôle et d'efficience des services impliqués dans la gestion des cas d'urgence. Par contre, la réunion des sources a permis de supprimer le bruit (entendons par là ce qui perturbe le flux de l'information) dans le processus et a apporté beaucoup de tranquillité aux organisations lors des situations critiques si propices au stress, et par conséquent une sensible amélioration dans l'efficacité des ressources disponibles. De sorte qu'il conviendrait d'ajouter aux prestations des SAIH celle de soutien psychologique et institutionnel des acteurs impliqués.

Les centres de contrôle sont donc devenus les noyaux du traitement de l'information, d'où l'on oriente les équipes de terrain vers les endroits véritablement problématiques, on leur fait des recommandations sur les opérations à effectuer, et on augmente ainsi leurs effets. De leur côté, les équipes se voient relayées dans les tâches de localisation et d'analyse des informations et peuvent ainsi se concentrer sur les opérations spécifiques qui leur incombent.

\section{- 3.2 Description de cas : les bassins rapides du Sud de l'Espagne}

Ce qui fait la spécificité du bassin sud de l'Espagne, du point de vue hydrologique, c'est la présence d'une chaîne de montagnes parallèle à la côte et proche de celle-ci, ce qui donne naissance à un réseau d'écoulement en peigne constitué de petits cours d'eau parallèles et en forte pente, et donc réagissant rapidement aux crues. Ajoutons à cela d'autres circonstances adverses comme l'invasion des plaines d'écoulement (déjà réduites) par l'activité économique ainsi que la présence de bassins de régulation à proximité de la côte, limitant les manœuvres de barrages. Par conséquent, toutes les opérations sont forcément très compromises.

L'Hozgarganta conflue avec le Guadiaro $7 \mathrm{~km}$, avant de se jeter dans la Méditerranée, à l'extrémité occidentale du bassin, près de Gibraltar. Il s'agit d'une des rares rivières du sud de la péninsule encore à l'état naturel. Avec un bassin de $359 \mathrm{~km}^{2}$, son cours ne présente pas de points conflictuels au long des 52 $\mathrm{km}$ qui vont de sa source jusqu'à la cote 1000 et n'inonde que des terres agricoles lors des plus grosses crues. Or le débit qu'il peut apporter au Guadiaro et les inondations que ce dernier peut provoquer dans son dernier tronçon en font un cas intéressant.

La coïncidence de certains facteurs (taille moyenne dans l'ensemble du bassin, stabilité de ses caractéristiques physiques et pluviométrie abondante) ainsi qu'une couverture hydrométrique acceptable grâce à un point de fermeture à 25 $\mathrm{km}$ de son embouchure et 3 stations pluviométriques dans le bassin, l'ont désigné comme bassin pilote pour l'étude des crues.

La figure 10 montre une représentation graphique des crues les plus importantes en termes de pluie moyenne dans le bassin (mm en ordonnée, à gauche) et hauteur limnimétrique de la nappe d'eau au point de fermeture du bassin (en mètres, à droite).

Ces contrôles ont servi au processus d'apprentissage et d'expérimentation d'un modèle du type réseau neuronal, qui permet de prédire le niveau pour les trois heures à venir (fig. 11), à partir de la pluviométrie estimée pour cet intervalle. Un deuxième modèle de transport donne les prévisions à l'embouchure, et permet de faire des pronostics pour les six heures à venir avec une précision suffisante pour prendre des mesures en ce qui concerne l'évacuation des populations.

\section{Résultats du modèle réseau neuronal.}




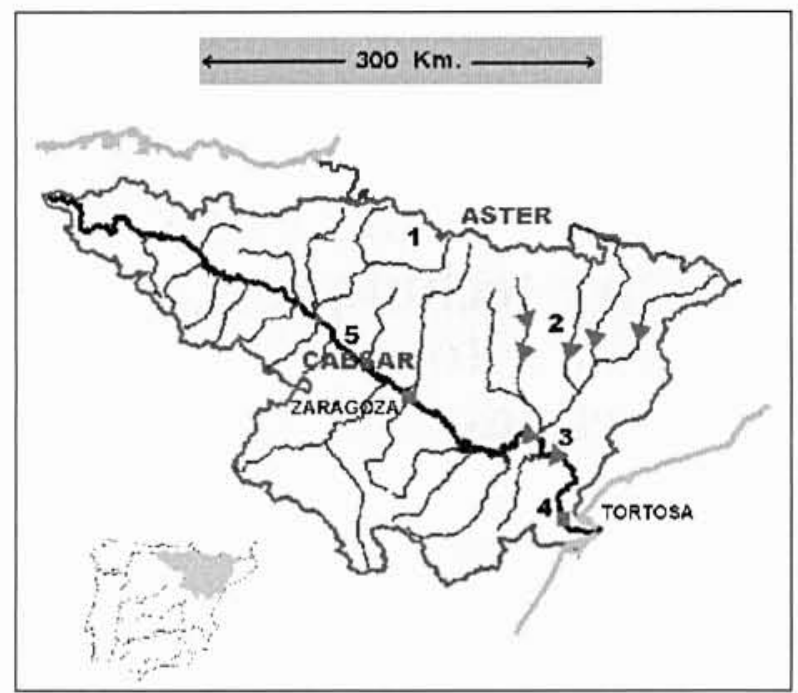

\section{Bassin de l'Ebre.}

\subsection{Description de cas : le bassin lent de l'Ebre}

Le bassin de l'Ebre, situé au nord-est de la Péninsule Ibérique et contigu à la France à hauteur des Pyrénées (fig. 12), est un bon exemple de gestion dans les bassins à réponse lente, puisqu'il comporte un collecteur général, l'Ebre luimême, qui rassemble toutes les eaux de ruissellement générées sur une étendue de $86000 \mathrm{~km}^{2}$.

Comme exemple significatif, nous avons choisi l'épisode survenu dans la semaine du 20 au 30 janvier 1997 enregistrant une crue assurément importante, où l'Ebre a atteint des débits dépassant $2700 \mathrm{~m}^{3} / \mathrm{s}$ près de son embouchure dans la Méditerranée.

En l'occurrence, le premier signal d'alarme a été donné grâce aux prévisions de débit de fonte des neiges fournies par le modèle pluvio-nival *ASTER. Les prévisions météorologiques pour les sept jours à venir faisaient s'attendre à une augmentation importante des apports fournis par les affluents pyrénéens de l'Ebre, étant donné l'important volume de neige accumulée à basses cotes, la montée probable des températures et la prévision d'importantes précipitations généralisées. C'est pourquoi on réalisa quelques premières manœuvres dans les barrages en amont du bassin en vue de renforcer la protection.

Le suivi de la situation effectué après par le SAIH de l'Ebre confirme l'accomplissement de ces prévisions, si bien qu'on décide de convoquer le Comité de Crises afin de faire face à la situation. Au vu de la généralisation des précipitations dans le bassin, la principale préoccupation se concentre sur la menace de débordement du fleuve à Saragosse et à Tortosa.

En ce qui concerne Saragosse, les estimations réalisées avec le modèle hydrologique de laminage CAESAR, confirment une faible montée des eaux dans cette ville: on a réalisé des prévisions sur le niveau de l'Ebre à son passage par Saragosse $\left(4,70 \mathrm{~m}\right.$, ce qui équivaut à $\left.2242 \mathrm{~m}^{3} / \mathrm{s}\right)$, anticipant sur plus de 30 heures, et postérieurement on a pu relever une erreur minime dans les prévisions $(7 \mathrm{~cm}$, pour une hauteur totale observée de $4,63 \mathrm{~m}$ ).

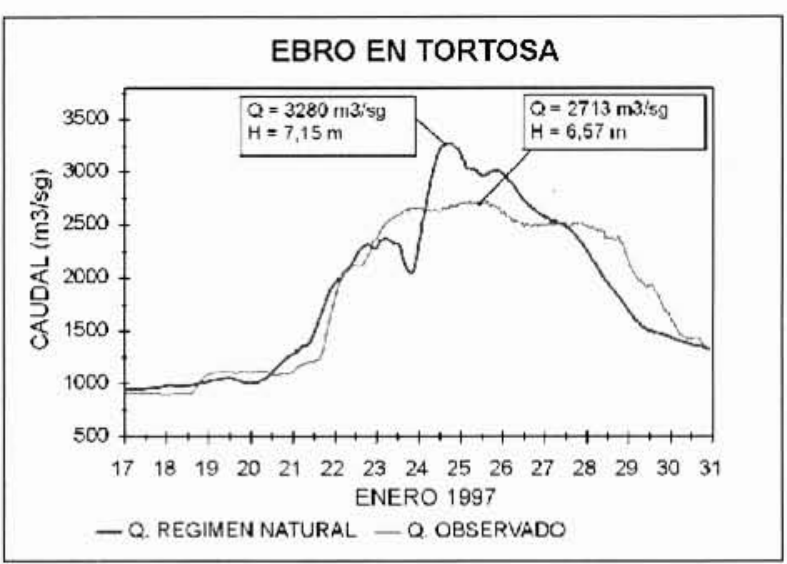

\section{Gestion de crue de l'Ebre - janvier 1997.}

La situation à Tortosa s'annonce beaucoup plus préoccupante, car on prévoit la conjonction de vagues de crue dans l'Ebre (celles provenant de la source, avec celles de la rive gauche provenant des Pyrénées). Il devient alors nécessaire de coordonner une action dans les principaux barrages du bassin, notamment ceux de Mequinenza $\left(1500 \mathrm{hm}^{3}\right)$, Yesa $\left(470 \mathrm{hm}^{3}\right)$, Mediano $\left(430 \mathrm{hm}^{3}\right)$, Oliana $\left(101 \mathrm{hm}^{3}\right)$, El Grado $\left(399 \mathrm{hm}^{3}\right)$ et Santa Ana $\left(236 \mathrm{hm}^{3}\right)$. Le schéma des opérations se résume en une première phase préventive de vidange des barrages inférieurs et de la rive gauche (Rivière Segre). Après, avec l'arrivée de la vague de crue par l'Ebre, ces deux ensembles de barrages seront chargés de 1) laminer la montée des eaux dans l'Ebre même (barrages inférieurs) et 2) de retenir les apports en amont des Pyrénées (région du Segre).

Le résultat de ce procédé de gestion des crues a évité que les débordements redoutés ne se produisent dans la ville de Tortosa (proche du delta de l'Ebre). On a enregistré dans cette localité un débit maximum de $2713 \mathrm{~m}^{3} / \mathrm{s}$, correspondant à une hauteur d'eau de $6,57 \mathrm{~m}$, qui laissait donc une marge de protection d'environ $30 \mathrm{~cm}$. A partir du calcul théorique des hauteurs d'eau maximales en régime naturel, on a estimé que sans cette intervention, le courant aurait atteint un débit de $3300 \mathrm{~m}^{3} / \mathrm{s}$, avec une hauteur de $7,15 \mathrm{~m}$, c'est-à-dire avec des débordements supérieurs à $25 \mathrm{~cm}$.

Le volume total de l'hydrogramme de la crue naturel s'élève à près de $3000 \mathrm{hm}^{3}$, ce qui représente environ la moitié de la capacité de l'ensemble des 57 principaux barrages existant dans le bassin et atteste l'importance de la crue enregistrée.

Ce mécanisme de gestion a quand même fait l'objet de critiques portant sur l'obligation même d'établir une certaine hiérarchie dans les objectifs à atteindre. Comme nous venons de le voir, la situation critique à Tortosa a forcé à réaliser des manœuvres préventives d'évacuation dans certains barrages, ce qui a provoqué l'inondation prématurée de plusieurs terrains agricoles en bordure de l'Ebre, qui, de toute façon, auraient été pareillement inondés. Cela entraîna les plaintes de quelques-unes des collectivités affectées, et a contraint à réaliser a posteriori une série d'études détaillées qui devaient justifier techniquement le bien-fondé des décisions adoptées en ces moments difficiles. 